\title{
Research Square \\ Experimental Study of Pore Permeability Characteristics Based On Variable-Mass System In Karst Collapse Pillars
}

\section{Mingkun Pang}

Xi'an University of Science and Technology

Tianjun Zhang ( $\boldsymbol{\nabla}$ tianjun_zhang@xust.edu.cn )

Xi'an University of Science and Technology

Rongtao Liu

Xi'an University of Science and Technology

Haotian Wang

Xi'an University of Science and Technology

\section{Research Article}

Keywords: sudden water management, permeability, particle loss, pore structure, seepage instability

Posted Date: January 13th, 2022

DOI: https://doi.org/10.21203/rs.3.rs-1235468/v1

License: (c) (i) This work is licensed under a Creative Commons Attribution 4.0 International License. Read Full License 
$1 \quad$ Article

\section{Experimental study of pore permeability characteristics based on 3 variable-mass system in Karst Collapse Pillars}

4 Mingkun Pang ${ }^{1,2}$, Tianjun Zhang ${ }^{1,2, *}$, Rongtao Liu ${ }^{2}$, Haotian Wang ${ }^{2}$

$5{ }^{1}$ Key Laboratory of Western Mine Exploitation and Hazard Prevention of the Ministry of Education, Xi'an, 6 China

$7{ }^{2}$ College of Safety Science and Engineering, Xi'an University of Science and Technology, Xi'an 710054, 8 China

\section{Abstract}

10 Particle loss is the root cause for the occurrence of Karst Collapse Pillars (KCP) sudden water events.

11 The pore adjustment of KCP filler will further induce seepage destabilization, and it is also a process

12 that sudden water catastrophe must go through. In order to investigate the direct relationship between

13 stress conditions, water pressure conditions, and gradation structure on the pore structure of rock

14 samples, the steady-state percolation method was used to investigate the percolation test system of

15 variable-mass crushed rock masses. The results show that: 1) the structural characteristics of rock

16 grains under the same stress environment are closely related to their extrusion fragmentation process

17 and the softening and scouring effect of water. Rubbing, rotating, fracturing, grinding and plugging are

18 the main forms of action of their intergranular action. 2) The filling particles before and after the loss

19 meet the fractal law and have fractal characteristics. 3) The percentage of fine particles in the whole

20 process of infiltration loss is as high as $34.4 \%$. The adjustment of pore structure is related to the

21 particle size gradation, and the reciprocal action of water flow will form a stable water-conducting

22 channel. 4) The sudden water process of the specimen under particle loss can be divided into three

23 stages: initial seepage, catastrophic destabilization and pipe flow surge.

24 Keywords: sudden water management; permeability; particle loss; pore structure; seepage instability

\footnotetext{
* Corresponding author: College of Safety Science and Engineering, Xi'an University of Science and Technology, 58, Yanta Mid. Rd., Xi'an 710054, Shaanxi, PR China (T. Zhang)

E-mail addresses: tianjun_zhang@xust.edu.cn, Tel:+86 13201827306 (T. Zhang)
} 


\section{Introduction}

When mining coal seams with complex hydrogeological conditions, groundwater usually coexists

27 with coal rock particles, and Karst Collapse Pillars (KCP) sudden water is the most common water 28 damage accident in coal mine production [1,2]. Ordovician tuff is a pressurized karst aquifer with

29 abundant water content and very high water pressure. Subjected to high pore pressure, groundwater

30 flows in the fractures and pores of fractured coal rock bodies, carrying small grains for transport and

31 loss [3,4]. Subjected to the corrosion and erosion of groundwater for a long time, the fine grains of the

32 coal rock body are lost with water and the structure will be gradually broken $[5,6]$. It seriously

33 deteriorates the internal structure of the rock mass and causes seepage hazards. It is important to study

34 the effect of rock mass loss on the seepage characteristics of rock masses to understand the engineering

35 properties of rock masses.

36 To study the pore seepage mechanism of crushed coal rocks in KCP, a large number of scholars

37 have conducted permeability test analysis. From the perspective of permeability tests, Kenney et al. [7]

38 investigated the migration of fine particles in the pore framework and used particle gradation curves to

39 determine the mass loss of fine particles. By establishing a finite difference model of particles, Sterpi et

40 al. [8] described the erosion transport mechanism of fine particles and analyzed the time-dependent

41 characteristics of fine particle density at various points within the effective seepage channel.

42 Meanwhile, Ma et al. [9] studied the effect of small particle transport on sudden water and the change

43 of hydraulic properties of granular sandstone. By designing the equipment and seepage experimental

44 system, Yao et al. [10] tested the seepage abrupt change law under different ratios and axial pressure.

45 Using the steady-state seepage method, Jie et al. [11] investigated the effect of axial stress on the

46 permeability coefficient of crushed rock masses. The non-Darcy flow characteristics of crushed rocks

47 are not only related to the porosity of crushed rocks, but also to the lithology of the rocks. Ma et al.[12]

48 tested the seepage characteristics of crushed mudstone, limestone and sandstone specimens with

49 different grain sizes at different porosity. Sulem et al.[13] investigated the hydrodynamic behavior of

50 sandstone specimens in relation to the surrounding pressure. Kong et al.[14] studied the relationship

51 between non-Darcy seepage parameters and the porosity and grain size of crushed rocks. By

52 establishing a fluid-solid coupling model, Yao et al.[15] solved the erosion and transport of particles in

53 karst trap columns under fluid action. There is also a part of researchers who are mainly interested in 
54 establishing the mechanism of sudden water, which in turn can reveal the formation and evolution

55 principle of sudden water in KCP. Zhang [16] presented the occurrence and possibility of sudden water hazard in the presence of aquifers in the upper or lower part of coal seams. Using numerical calculation methods, Yin et al. [17] carried out a simulated coal mine mining scenario under the action of both KCP holes and pressurized water. Using the rock mechanics principle, Wan et al. [18] described the path of high-pressure water from the aquifer gushing out to the working face. Based on elastic damage theory, Zhu et al. [19] established a damage based hydraulics model to simulate the emergent water behavior of mining under the action of faults and KCPs. Based on seepage theory and groundwater dynamics theory of porous media, Hui et al. [20] established a flow-solid coupling model for karst trap columns. Obviously, mud rocks are prone to mudification and disintegration when they encounter water, which leads to the formation of crushed coal rock bodies of different shapes. This can lead to great differences in mechanical properties and permeability between fractured and intact coal rocks.

The above studies mainly discuss the steady-state percolation law of this porous medium composed of broken coal bodies under certain conditions. However, in the percolation process of KCP, the fine particles are washed by water and the porosity of KCP changes, so the water percolation in $\mathrm{KCP}$ is a variable-mass system. In this study, we start from the perspective of variable mass percolation, and further observe the changes of grain structure of coal rock body before and after percolation by variable mass percolation test. The effect of stress conditions on the pore structure of broken coal rock is explored, the principle of the action of pore water pressure on the crushing of coal rock body grains is analyzed, and finally the process of pore damage leading to disaster occurrence in $\mathrm{KCP}$ is also given.

\section{2. Theoretical background}

2.1 Variable mass sudden water model with fluid-solid coupling

The main component of the KCP fill is the fractured coal rock body. This structure can penetrate through the Ordovician tuff, mudstone, and coal layers [21]. When the pressurized water pressure within the Ordovician aquifer is greater than the principal stress of the key layer, the destruction of the column within the surrounding high permeability region will occur [22-24]. At this stage, many scholars mainly use series of sudden water models, and the lithological distribution perspective to

81 precisely analyze the sudden water mechanism. 
It is assumed that the infiltration process is consistent with Darcy flow and the fluid medium is

83 incompressible. The diffusion of particles in the fluid can be neglected, and Yao et al. [10] gave the

84 mass conservation equation for coal grains as:

$$
\frac{\partial(1-\phi)}{\partial t}+\nabla \cdot\left[(1-\phi){\stackrel{\mathrm{v}}{v_{s}}}^{\mathrm{r}}\right]=-\frac{n \& \mathrm{k}}{\rho_{s}}
$$

85 Where: $v_{s}$ denotes the percolation rate; $m$ denotes the mass; $\rho$ denotes the fluid density. The equation of motion of the grain is given by:

$$
\rho_{p} \frac{\pi d_{p}^{3}}{6} \frac{\partial u}{\partial t}=C_{D} \frac{\pi d_{p}^{2}}{4} \frac{\rho_{f} u^{2}}{2}-\frac{\pi d_{p}^{3}}{6} g\left(\rho_{s}-\rho_{f}\right)
$$

The equation for the evolution of the pore space of the crushed coal and rock body is:

$$
\frac{\partial \phi}{\partial t}= \begin{cases}\lambda\left(\phi_{\max }-\phi\right) \mathrm{C} q_{f} & \left(v_{f z}>u_{t}\right) \\ 0 & \left(v_{f z}<u_{t}\right)\end{cases}
$$

88 Where: $\phi$ denotes porosity; $\lambda$ denotes infiltration length; $q_{f}$ denotes flow rate; $u$ denotes critical 89 percolation rate.

$90 \quad 2.2$ Theoretical model of permeability of trap columns under erosion

Based on the study of Sakthivadivel [25], the effect of erosion on the permeability of fractured rock masses was considered.Zhang et al. [26] used both permeability tests and numerical calculations to summarize the equations controlling the pore evolution of porous media in fractured coal bodies

94 under erosion.

$$
\frac{\partial \phi}{\partial t}=\lambda_{1} \rho_{0}\left(\phi_{\max }-\phi\right) c\|q\|
$$

Assuming that the pore-fissure dual medium evolution process of the crushed coal rock body is similar, based on the relevant theory [27,28], Elsworth gave the equation for the fissure evolution of the

97 coal rock body under the erosive action of the porous medium with particle migration, where the 98 permeability of the unit body is as follows.

$$
k=k_{m_{0}}\left(\frac{\phi_{m}}{\phi_{m_{0}}}\right)^{3}\left(\frac{1-\phi_{m_{0}}}{1-\phi_{m}}\right)^{3}+\frac{b^{3}}{12}
$$

99 Where: $\phi$ denotes specimen porosity; $t$ denotes time; $b$ denotes percolation characteristic length. 
101 Using the permeability test method of pore structure, the trapped column sudden water test with

102 different bearing permeability history under the effect of particle migration was carried out by changing

103 the bearing history. In simulating the sudden water behavior of $\mathrm{KCP}$, different stress paths and

104 infiltration stages are used to describe the general phenomenon of KCP sudden water. By summarizing,

105 the elements affecting the water-conducting fractures of the $\mathrm{KCP}$ and the damage characteristics of the

106 structure are analyzed.

$107 \quad 3.1$ Materials

$108 \quad$ (1) Research Background

109 HEYANG COAL MINE is located in Shaanxi Province and the well field is in the eastern part of

110 the Weibei coalfield. The design production capacity of Heyang coal mine is $1.2 \mathrm{Mt} / \mathrm{a}$. $5 \#$ coal seam is

111 mined with the original design of comprehensive mechanized topping coal mining. 5\# coal seam has

112 original moisture of $0.76 \%$ and bad lumpiness. 5\# coal seam is a typical "three soft" coal seam, the top

113 plate is unstable and easy to fall, the coal seam is soft, the solidity coefficient is small and easy to flake,

114 the bottom plate is soft, the compressive strength is low and easy to bottom. The immediate roof of the

115 5\# coal seam is gray-black siltstone and sandy mudstone. The main roof is gray fine-grained sandstone,

116 brittle and easily broken. The main floor is gray K3 quartz sandstone or siltstone, containing mica

117 fragments, with more developed fissures.

$118 \quad$ (2) Material preparation

119 The specimen was selected from the dark gray mudstone of HEYANG COAL MINE in Shanxi

120 Province. The hydrogeological conditions of this mine are complex, and there is a possibility of water

121 breakout in the water-rich abnormal area at the bottom of the coal seam during back mining. The core

122 density of the mudstone was calculated to be $\rho=2546 \mathrm{~kg} / \mathrm{m}^{3}$, and the crushed rock samples selected from

123 the working face before the test were screened by BZS-200 standard vibrating sieve machine according

124 to the actual 7 particle size ranges of $0 \sim 2.5 \mathrm{~mm}, 2.5 \sim 5 \mathrm{~mm}, 5 \sim 8 \mathrm{~mm}, 8 \sim 10 \mathrm{~mm}, 10 \sim 12 \mathrm{~mm}, 12 \sim 15 \mathrm{~mm}$

125 and $15 \sim 20 \mathrm{~mm}$. The physical samples of crushed particles in each size range are shown in Figure 1. 


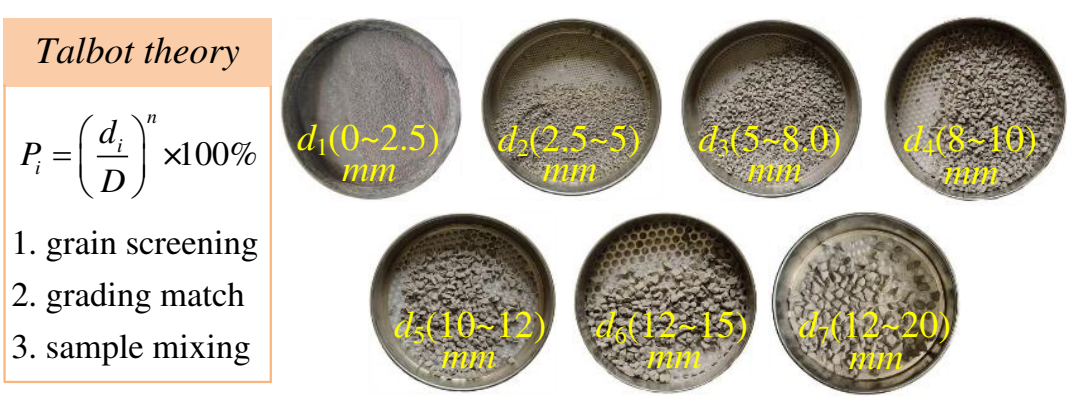

Fig. 1 Physical crushed granular rock samples in each particle size interval

128 The crushed grains in each grain size interval were obtained by screening. Using Talbot gradation 129 theory [29], the mass share of each interval can be calculated and then mixed to obtain the crushed 130 specimens. The Talbot gradation equation is shown in Eq. 6.

$$
P_{i}=\frac{M_{d}}{M_{t}}=\left(\frac{d_{i}}{D^{*}}\right)^{n} \times 100 \%
$$

131 Where: $P_{i}$ is the proportion of mudstone particles with diameter less than $d_{i} ; d$ is the diameter of 132 mudstone particles, $\mathrm{mm} ; D^{*}$ is the maximum diameter of mudstone particles, $\mathrm{mm} ; n$ is the Talbot 133 power index. Four kinds of mixed crushed rock samples with gradation values of $n=0.2, n=0.4, n=0.6$ 134 and $n=0.8$ were prepared, and three groups of $1800 \mathrm{~g}$ each. The mass distribution of each grain size of 135 the samples is shown in Table 1.

Table 1 Mass distribution of each particle size interval of the specimen

\begin{tabular}{cccccccc}
\hline \multirow{2}{*}{$\begin{array}{c}\text { factor } \\
n\end{array}$} & $0 \sim 2.5$ & $2.5 \sim 5$ & $5 \sim 8$ & $8 \sim 10$ & $10 \sim 12$ & $12 \sim 15$ & $15 \sim 20$ \\
\cline { 2 - 7 } & 0.5 interval of each particle size $d_{\mathrm{i}} / \mathrm{mm}$ \\
\hline 0.2 & 1187.6 & 176.6 & 134.5 & 68.4 & 58.2 & 74.2 & 100.6 \\
0.4 & 783.5 & 250.3 & 213.8 & 116.5 & 103.2 & 137.0 & 195.7 \\
0.6 & 516.9 & 266.6 & 255.3 & 148.8 & 137.3 & 189.8 & 285.4 \\
0.8 & 341.0 & 252.7 & 271.0 & 169.1 & 162.4 & 233.8 & 370.1 \\
\hline
\end{tabular}

137 3.2 Methodologies

$138 \quad$ (1) Experiment design

139 Set 10,15, 20, 35 and 40KN, 5 levels of axial increasing load. For the loading method, ordinary 140 hydraulic oil with density $\rho$ of $874 \mathrm{Kg} / \mathrm{m} 3$ and dynamic viscosity of $1.96 \times 10-2 \mathrm{~Pa} \cdot \mathrm{s}$ was selected as the 141 permeate, and four axial stresses of 3MPa, 4MPa, 5MPa, and 6MPa were set respectively, with loading 142 time of $120 \mathrm{~s}$ and holding time of $15 \mathrm{~min}$ for all axial stresses at each level. MPa, 1.5MPa, 2.0MPa and $1432.5 \mathrm{MPa}$ were set for percolation. 
145 Commission the press and start the test according to the preset loading method. (3) Record the

146 displacement under each level of axial stress. (4) Start the hydraulic pump for controlling the osmotic

147 pressure, and apply $0.5,1,1.5,2$ and $2.5 \mathrm{MPa}$ of osmotic pressure at each level of loading axial stress

148 in turn by adjusting the valve. (5) The flow rate of each osmotic pressure gradient was weighed and

149 recorded, and the magnitude of permeability was determined by the flow rate. (6) After each completed

150 measurement of one specimen, its stress-strain data were obtained through the computer data

151 acquisition system, and then the previous steps were repeated for other specimens, and each set of data

152 was recorded until the test was completed.

(2) Experimental equipment

There will be a small amount of sediment gushing out before the burst water of $\mathrm{KCP}$, and the sediment gushing out means the migration and mass loss of fine particles in the trap column fill, the essence of this phenomenon is the problem of variable mass percolation in the crushed rock body under the migration of particles. In order to study the influence of the gradation composition, bearing history and infiltration stage of the trap column fill on the migration of particles, infiltration pore space and the destruction characteristics of the sudden water channel under different burial depths, the variable-mass

160 infiltration test apparatus was designed by ourselves. The broken rock body variable-mass percolation test system is shown in Figure 2.

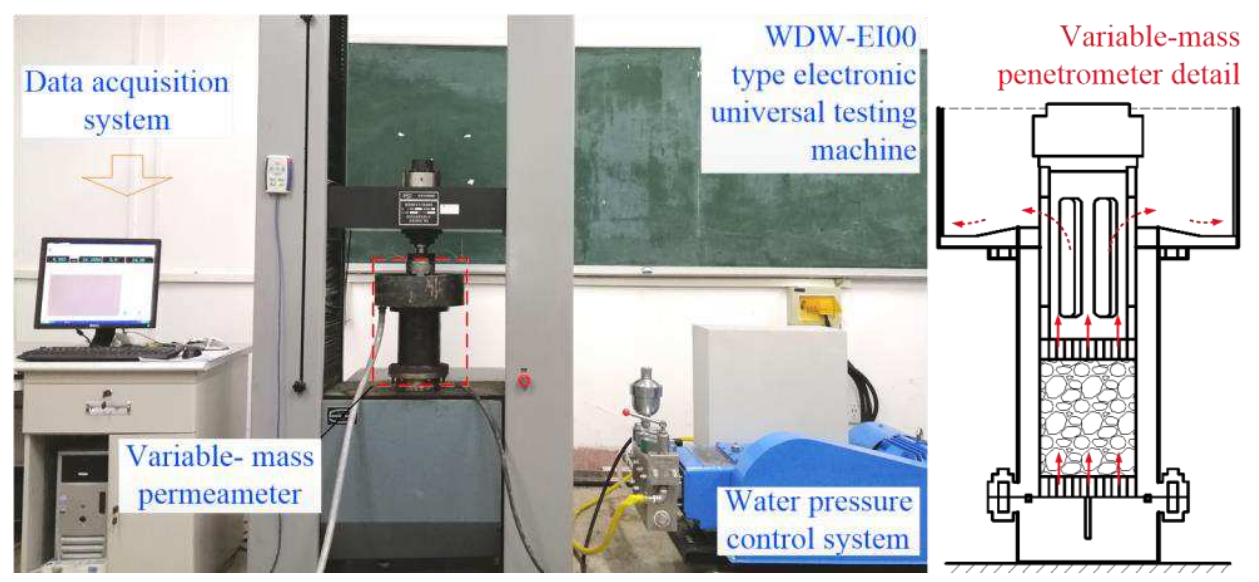

Fig. 2 Variable-mass percolation test system for crushed rock masses 
166 recovery device. Among them, the infiltration water pressure supply device is the main improvement 167 part of this test, which is composed of SY-40 high-pressure plunger pump, accumulation tank and sensors, etc. It can provide infiltration water pressure with rated pressure of $30 \mathrm{MPa}$ and flow rate of

$1690.6 \mathrm{~m}^{3} / \mathrm{h}$. The pressure and flow rate values can be adjusted stably and collected in real time at the 170 corresponding moment, and can be used to simulate the pressurized water pressure at the bottom of the trap column under different burial depths.

\section{4. Results and Discussions}

173 For the sudden water problem of water-rich conductive trap column under particle migration, the 174 pore penetration test considering the grading structure, bearing history and penetration stage was carried out by using the improved particle migration percolation test system.

4.1 Changes in the structural characteristics of particles before and after infiltration

According to the fugitive state of the specimen before and after infiltration, representative rock sample images were selected and the macrostructure and microscopic characteristics of the crushed rock samples before and after infiltration were obtained by using EM-30AX type scanning electron microscope (SEM).

181 (1) The state of the specimen before penetration

182 Before the test, stresses were transferred between rock sample particles in the form of point-to183 point/face contacts, resulting in large size crushed rock masses being able to transfer energy or stress to 184 adjacent rock masses through more contacts. For mixed size rock masses, the larger the rock mass 185 particles, the more contact points/faces corresponding to the particles, the less likely to be broken. The 186 microscopic characteristics of the broken rock sample before penetration is shown in Figure 3.

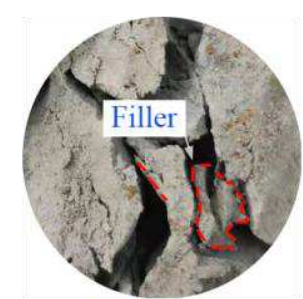

(a) Extrusion fragmentation

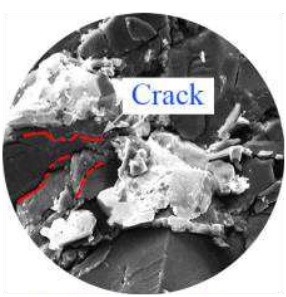

(b) Grain crushing $500 \times$

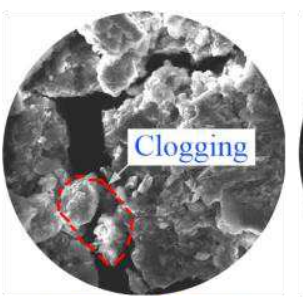

(c) Channel blockage $1000 \times$

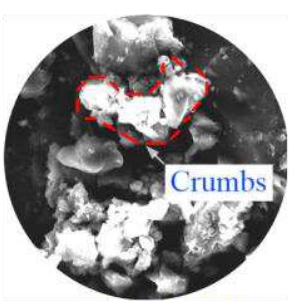

(d) Chip flaking $1000 \times$

Fig. 3 Microscopic characteristics of broken rock samples before infiltration 
190 more contact points of large particles and contact points of smaller particles are not only related to the contact points, but also related to the contact state between the particles, rubbing, rotation, fracture,

192 grinding and filling constitute the general form of contact action between the particles. In the 193 microscopic specimen surface structure, the inter-particle pore structure is significantly reduced, and the fracture is continuously filled with fine particle debris. New fractures are continuously formed by

195 the rock particles under load, and the fine particles around these fractures overcome frictional resistance and undergo relative sliding or dislocation. Filling and plugging the storage space and transport channels in the process of stress damage, and then reach a new equilibrium position, forming a more compact and stable structure.

After the test, the rock block particles are scoured and softened by the water flow, which makes the original fine particles in the rock sample of the filling migrate, and in this process collide and grind the larger diameter particles and appear new secondary fine particles. The process destroys the granular liquid film near the surface of the rock mass, and there are obvious cracks and bonding phenomena on

204 the surface of the destroyed particles, and they contain granular or powdery debris materials. The microscopic characteristics of the broken rock sample after penetration are shown in Figure 4.

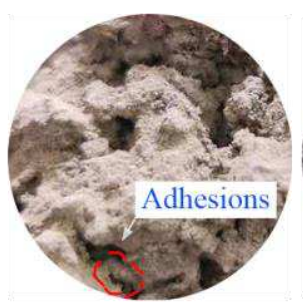

(a) Grain after penetration

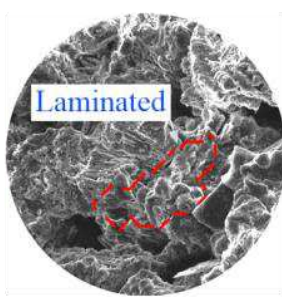

(b) Laminar structure $300 \times$

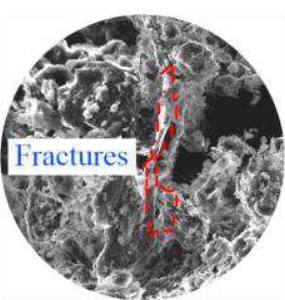

(c) Crushing structure $500 \times$

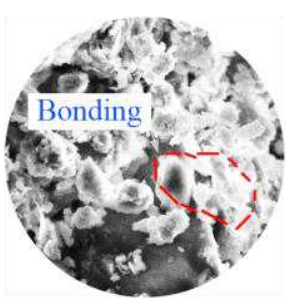

(d) Grain adhesion $1000 \times$

Fig. 4 Microscopic characteristics of broken rock samples after penetration

In the microscopic view, a large number of intergranular features such as weak cementation,

209 lamination and fragmentation can be observed after the rock mass particles are magnified by 500 times.

210 The occurrence of the above-mentioned features in the rock sample is not only related to the extrusion

211 and fragmentation between the particles, but also to the softening and erosion of the particles by water.

212 The two together act on the rock mass filling inside the trap column, causing the basic structural 213 morphological features of the rock sample particles before and after infiltration. 
$214 \quad 4.2$ Effect of axial loading on the pore structure of specimens

215 Porosity is a key parameter in determining the permeability characteristics of crushing media [30].

216 Therefore, it is of great engineering value to study the variation law of porosity of the crushed coal

217 body during the graded loading process. The pore structure of the crushing medium is changed by the

218 gradual increase of the stress. In the test, the porosity parameter of the test can be calculated by

219 calculating the corresponding displacement.

$$
f=1-\frac{m}{r_{1} \mathrm{~A}(h-\Delta h)}
$$

220 Where: $m$ is the mass of the coal sample in the cylinder; $h$ is the initial height of the coal sample; $\rho_{1}$ is

221 the density of the coal body; $\Delta h$ is the collected axial displacement; $A$ is the cross-sectional area of the 222 cylinder.

223 (1) Porosity change pattern

224 The porosity decreases with the increase of axial stress, and the larger the axial stress, the slower 225 the decrease. The porosity decreases rapidly when the axial stress is from $3 \mathrm{MPa}$ to $5 \mathrm{MPa}$ and 226 decreases gradually after loading to $5 \mathrm{MPa}$. As a general rule, the porosity of crushed specimens shows 227 a negative exponential trend with the increase of axial stress. In order to obtain the relationship 228 between axial stress and porosity of the crushed coal rock mass, corresponding experiments were carried out to determine the relevant parameters. The porosity-axial stress curves of each group of 230 specimens are shown in Figure 5.

231

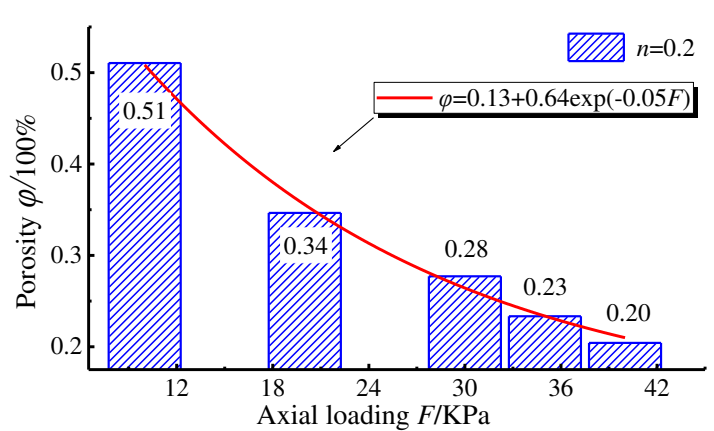

(a) sample $n=0.2$

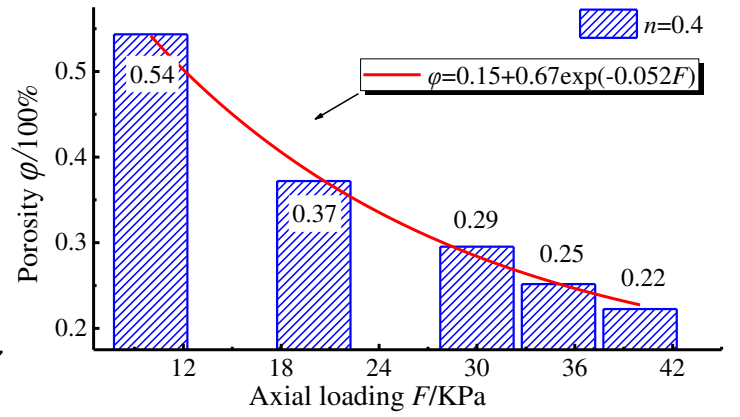

(b) sample $n=0.4$ 


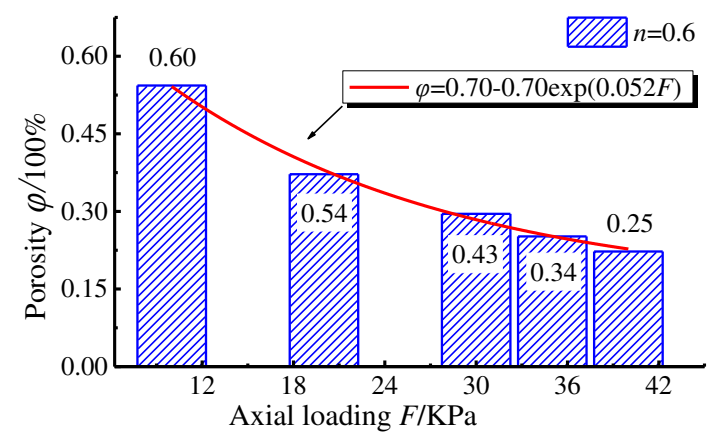

(c) sample $n=0.6$

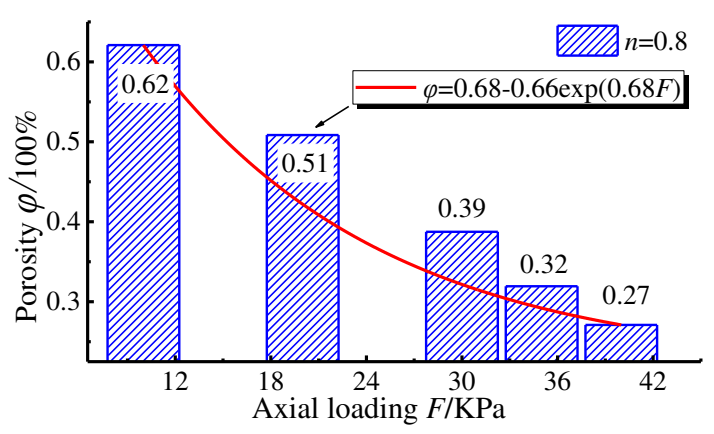

(d) sample $n=0.8$

Fig. 5 Porosity-axial stress curve of each group of specimens

It can be analyzed that the porosity decreases gradually with the increase of load under different

235 bearing history and permeability stages. The whole porosity change process can be divided into three 236 stages: gradual compacting, rapid adjustment and relative stability. In the gradual compacting stage,

237 there is particle migration behavior and pore adjustment magnitude is larger under changing bearing 238 history, while in different permeability stages, particle migration behavior is weaker, and the pore 239 adjustment magnitude of specimens with gradation value $n=0.2$ and $n=0.4$ is much higher than the pore 240 adjustment magnitude of other specimens. In the rapid adjustment stage, the particle migration 241 behavior of the specimens with $n=0.2$ and $n=0.4$ is obvious due to the load surge behavior under 242 different bearing histories. The relative stability phase generally occurs between the 4th and 5th load 243 levels, between $35 \mathrm{KN}$ and $40 \mathrm{KN}$. After adjusting the infiltration stage, the porosity is more dense, with 244 values mostly between 0.448 and 0.531 . This indicates that there is better consolidation between the particles of the specimens after adjusting the infiltration stage.

(2) The law of the change of the lost mass

During groundwater softening and erosion, migration of fines originally present in the rock samples of the fill occurs. During migration, the collision and fragmentation of larger diameter particles results in the emergence of secondary particles [31]. In the case of particle loss, the porosity of each sample is:

$$
\varphi_{i}=\varphi_{0}+\frac{1}{\pi \mathrm{a}^{2} h \rho_{m}}\left(\Delta m_{1}+\Delta m_{2}+\cdots+\Delta m_{i}\right)
$$

In the test, the use of electronic scales to obtain the infiltration quality, the collection interval of 10 obtained at a certain time of flow increase. 


$$
v_{t=k}=\frac{m_{k}-m_{k-1}}{\rho_{w} \pi a^{2} \Delta t}
$$

254 Where: $m_{k}$ is the mass of water out; $\rho_{w}$ is the mass density of water; $a$ is the radius inside the cylinder;

$255 \Delta t$ is the sampling time interval. The relationship between the loss mass distribution of each group of 256 specimens is shown in Figure 6.

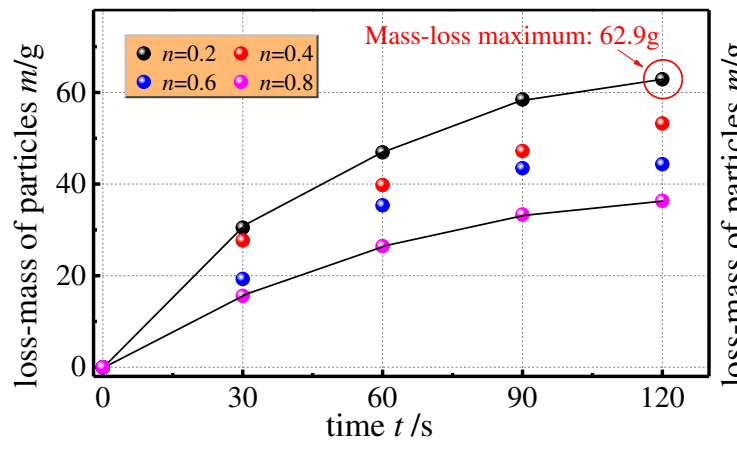

(a) First stage load $10 \mathrm{KN}$

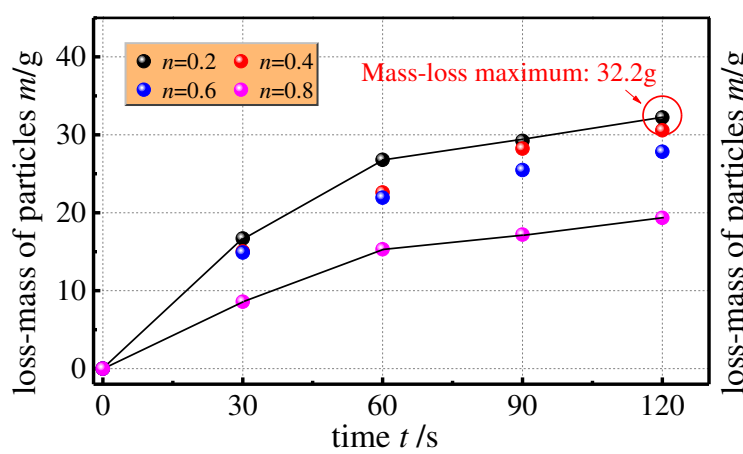

(c) Third stage load $30 \mathrm{KN}$

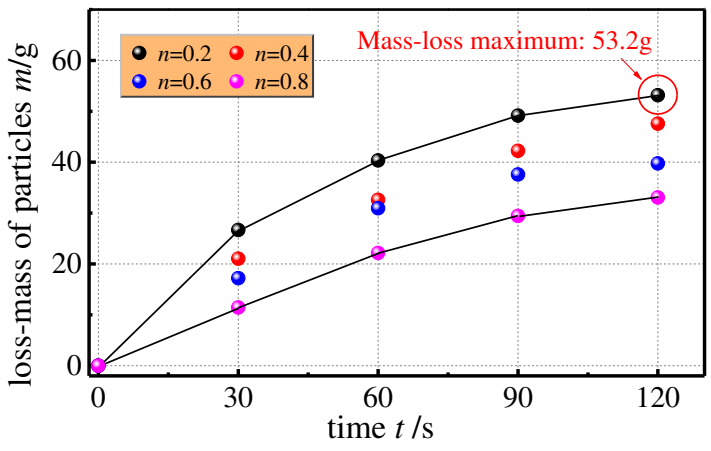

(b) Second stage load $20 \mathrm{KN}$

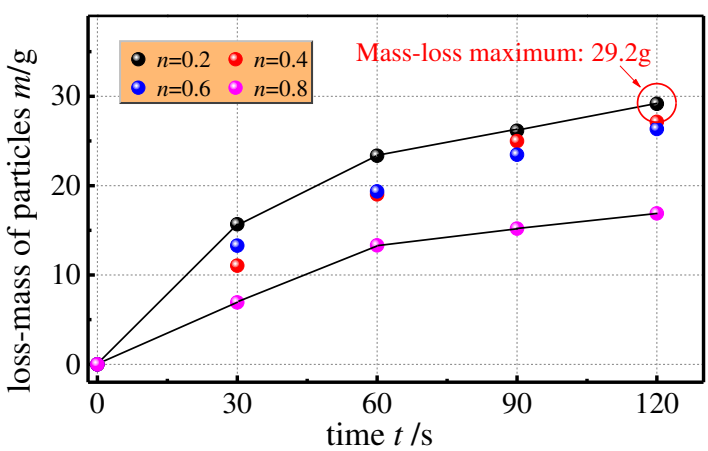

(d) Fourth stage load $40 \mathrm{KN}$

Fig. 6 Relationship between the loss mass distribution of each group of specimens

We measured the permeation parameters of each specimen through the experiment, and the loss mass of each group showed an increasing trend with time and then a flat trend. The maximum values of mass loss were $62.9 \mathrm{~g}, 53.2 \mathrm{~g}, 32.2 \mathrm{~g}$ and $29.2 \mathrm{~g}$, respectively, and the maximum value of mass loss was

263 found in the first group $(n=0.2)$ with the maximum value of $62.9 \mathrm{~g}$, indicating that the fine structure of

264 the specimen started to change and the coal body expanded outward, while the porosity of the broken coal body increased, resulting in a larger pore throat inside the specimen and thus the permeability of

266 the broken coal body became larger. The fine particles produced during the flushing process repeatedly erode the larger diameter particles. The mud continues to erode the fines and soften the newly exposed surface. This in turn leads to adjustments in the permeable pore space of the KCP and a shift in seepage patterns. Ultimately, this leads to sudden water incidents. 
4.3 Relationship between pore water pressure and particle damage in the state of stress between the grains of the coal rock body. It accelerates the expansion of the original cracks in the rock mass, reduces the strength of the grains in the rock mass, and causes the fracture form of the rock mass to become more complex.

In order to study the deformation characteristics of crushed coal grains after compaction. By collating and processing the test data, we obtained the characteristics of the grain size distribution of each group of specimens. The particle size distribution pattern of each specimen after destruction is shown in Figure 7.

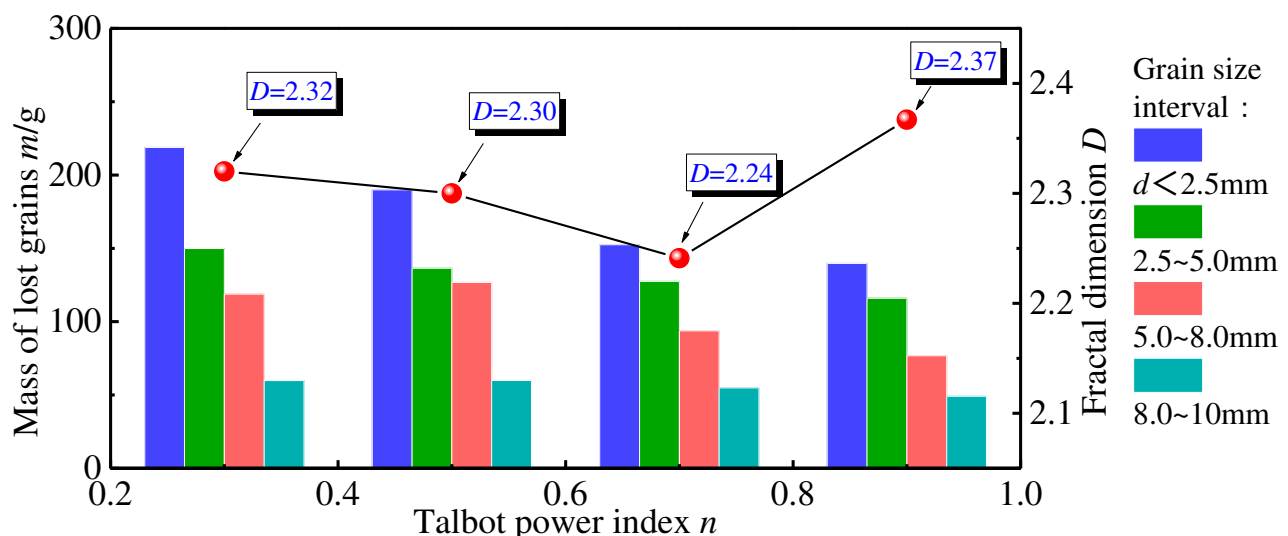

Fig. 7 Particle size distribution pattern of each specimen after destruction

The mass $\mathrm{m}$ of siltation particles decreases with increasing Talbot power index $\mathrm{n}$. The distribution of siltation particles for each grade distribution is dominated by the particle size in the interval $0-2.5$ $\mathrm{mm}$. We can observe the loss of particles at all levels of the matched sample, and further statistics were obtained for the fractal dimension of the particle size distribution. The values are $2.32 \mathrm{~g}, 2.30 \mathrm{~g}, 2.24 \mathrm{~g}$ and $2.37 \mathrm{~g}$ respectively. This indicates that the specimens with the graded value of $n=0.2$ are more likely to silt more fine particles, and the large particles in the specimens weaken the hydraulic capacity of the graded specimens. This indicates that under the high stress, the fine internal structure of the coal body starts to change and the coal body expands outward, while the porosity of the broken coal body

290 increases, resulting in larger pore throats inside the specimens, which makes the permeability of the broken coal body larger and more permeable. 
At high pore pressures, groundwater can seep and flow into fractures and pores, carrying small

294 particles with it to create transport and loss phenomena [31]. Some fine-grained material moves with water flow and part of it is lost, changing the structure and strength, grain size distribution (GSD), local stress field, and seepage field of the crushed rock mass. To represent the change in GSD during seepage, a new physical quantity, residual mass ratio, was defined.

$$
R_{r}\left(d_{i}\right)=\frac{m_{r}\left(d_{i} \leq d \leq d_{i+1}\right)}{m_{o}\left(d_{i} \leq d \leq d_{i+1}\right)} \times 100 \%
$$

where: $m_{r}$ denotes the original mass; $m_{o}$ denotes the remaining mass; and denotes the original mass
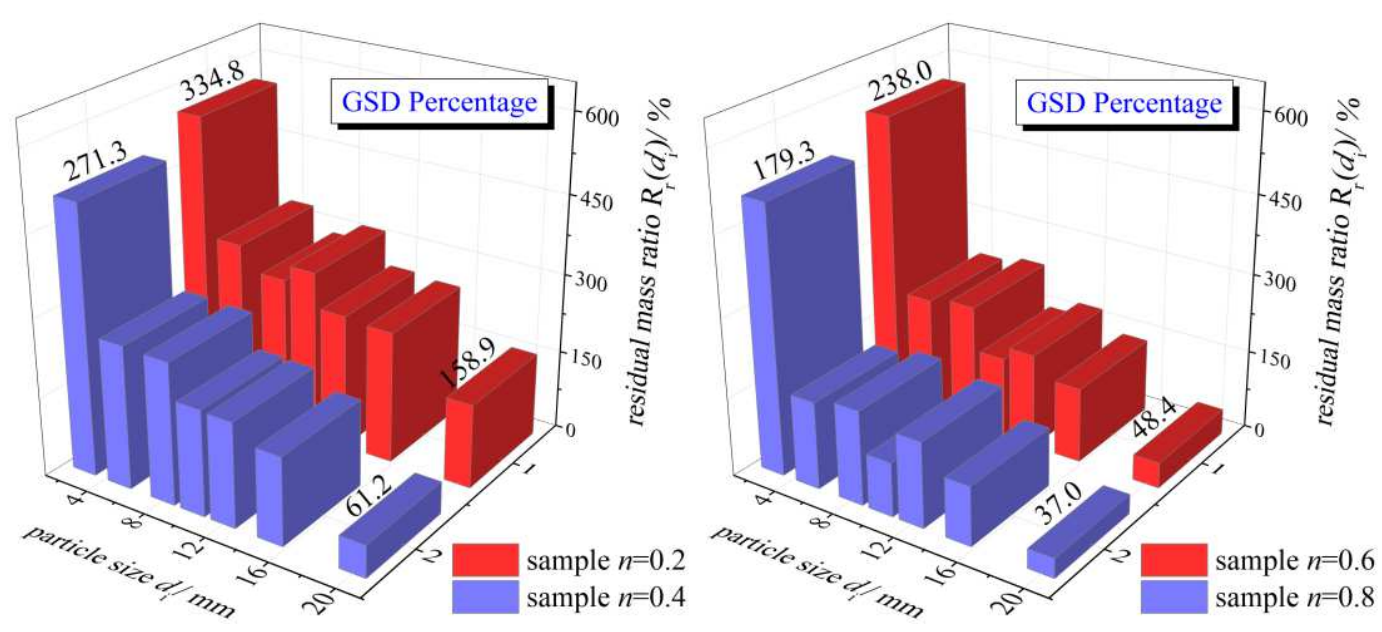

Fig. 8 Distribution pattern of GSD values for each group of specimens

The data show a surge in the percentage of small particles in all groups of specimens, with the particles (less than $2.5 \mathrm{~mm}$ ) in the specimens of group $n=0.2$ is up to 3.35 times. The fourth group $(n=0.8)$ had a small increase in small particles of only 1.79 times. We believe that during the seepage process, the broken grains squeeze and rub against each other under the pore pressure and the larger rock masses are destroyed. It produces new secondary material, finely broken rock masses are transported with water flow, and some rock masses are washed out of the cylinder, thus forming the change of GSD. 
312 4.4 Analysis of the evolution of pore disaster-causing processes in trap columns

313 (1) Change of pore structure during water suddenness

314 The occurrence of KCP sudden water behavior is mostly characterized by short duration and

315 sudden occurrence. The sudden water process will appear obvious water spouting behavior (a), at this

316 time the fill of fine solid particles constantly migrate with the water flow, the roadway working surface

317 will have obvious particle siltation phenomenon, which has been revealed through the indoor test with

318 the tray (b). With the continuous osmotic water pressure, so that the volume fraction of solid particles

319 in the pore space of the filling is gradually reduced, the more obvious collapse cracks will appear in the

320 sudden water channel (c). The infiltration characteristics of the sudden water process are described as

321 shown in Figure 9.

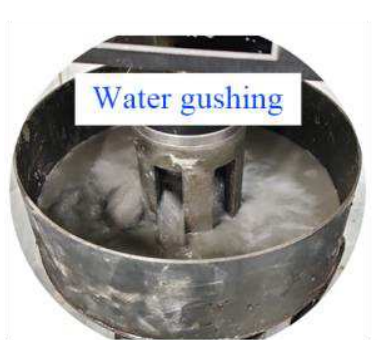

(a) Stage I of the sudden-water

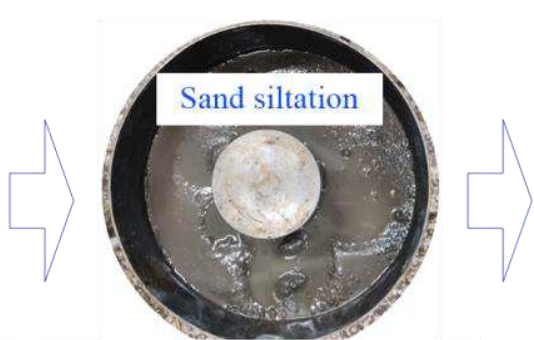

(b) Stage II of the sudden-water

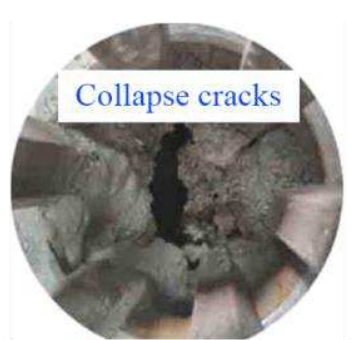

(c) Stage III of the sudden-water

Fig. 9 Description of the infiltration characteristics during the sudden water flow

The occurrence of sudden water accidents in the trap column is mostly due to the tectonic stress around the column, mining stress and strong seepage pressure off. With the change of pore water pressure, the primary or secondary fine particles in the filling pore with the water flow is constantly migrated out, in the process of permeability pore expansion, permeability increase hair over to affect the filling column body seepage velocity and particle carrying capacity, followed by more fine particles to transport until the remaining skeleton and difficult to migrate the stubborn parent particles, secondary porosity is relatively stable, the influx of water tends to level off. Such an alternating effect of the transformation process, it can be concluded that: the migration of the filling particles is the inherent nature of the trap column sudden water behavior.

There are three states of motion in the infiltration process: deformation of the granular structure,

335 infiltration of water under high pressure and migration of particles. When these three types of motion 336 fulfil a certain condition, they cause a sudden change in the pore structure of the KCP fill. It accelerates 
337 the rate of particle migration and widens the pore space of the fill. The process of KCP pore 338 catastrophe is shown in Figure 10.

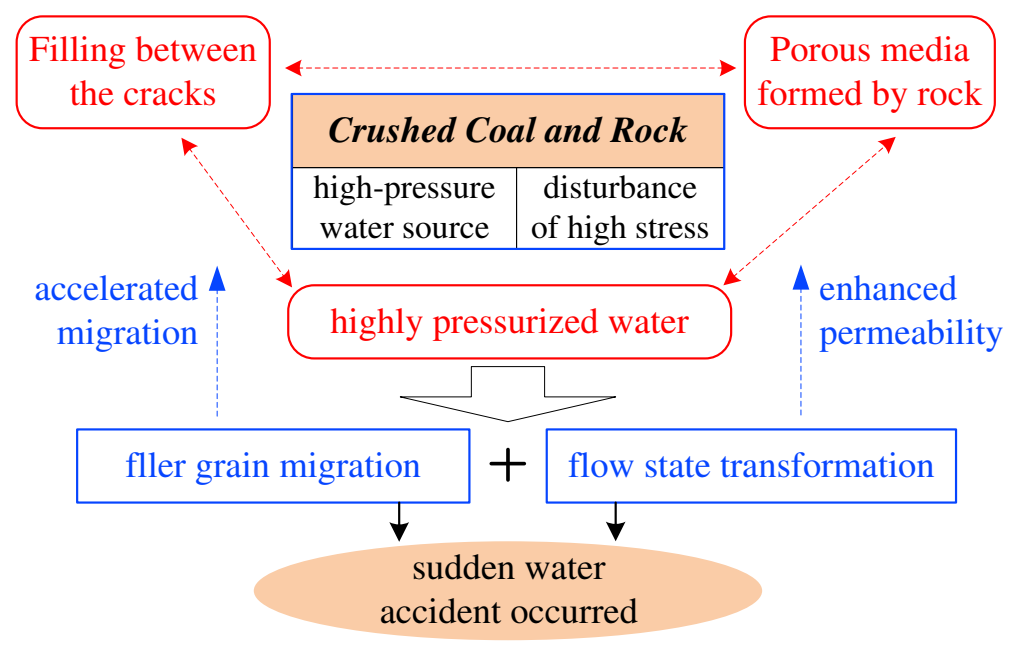

Fig. 10 Schematic diagram of the process of KCP pore catastrophe action

The analysis shows that the volume fraction of solid particles in the pores of the filler block is 342 reduced by the osmotic pressure of the water. The rupture of the fluid film on the surface of the 343 dissolving rock mass causes the filler particles to migrate into the pore space and migrate with the fluid.

344 This leads to an increase in the porosity of the fractured rock mass. In this way, the original particles in 345 the pore space and dissolution of the particles produced by continuous migration out with the water 346 flow, the permeability of the broken coal body is increasing; permeability increases in turn increase the 347 water flow speed and carrying capacity, so that more particles migrate loss, such an interactive process 348 of changing quality, and constantly add the permeability of the broken coal body, until the remaining 349 skeleton and difficult to migrate the filler particles, porosity no longer increases, and the water volume 350 is stabilized. The influx of water tends to stabilize.

351 There are four stages in the extrusion and fragmentation destruction process of particles: (1) the 352 existence of weak contact; (2) dislocation slip; (3) occlusion; (4) compression density, the larger the size 353 of the rock mass, the stronger the extrusion and fragmentation that can be withstood; the general 354 disclosure process of the erosion and scouring effect of pressurized water also includes four stages: (1) 355 the destruction of the liquid film on the surface of the specimen particles; (2) the softening of the 356 surface of the newly exposed particles; (3) the adjustment of the infiltration pores of the filling; (4) and 
357 the transformation of the seepage form. Therefore, the migration of fill particles is the key factor to

358 trigger the sudden change of rock seepage.

\section{5. Conclusions}

360 Based on the granular characteristics of the fill samples before and after infiltration, the structural 361 properties of the fill particles are observed. Combined with the performed pore penetration tests of 362 KCP filler with different grading structures and the sudden water penetration tests with different bearing penetration histories. The following conclusions were mainly drawn.

1) The structural characteristics of the rock masses are mainly related to the crushing of grains and 365 the action of water on scouring. Under the same stress environment, the structural characteristics of rock grains are closely related to the crushing and fragmentation process and the softening and scouring effect of water, and the general contact processes of rolling, rotating, fracturing, grinding and plugging are the general contact processes.

2) The percentage of fine particles in the process of mass loss can increase to 3.35 times. During compaction and deformation of the crushed coal body, porosity decreases as the axial stress increases. The bearing capacity also increases as the porosity decreases, with the porosity decreasing rapidly as the axial stress increases from $3 \mathrm{MPa}$ to $4.5 \mathrm{MPa}$. After loading to $5 \mathrm{MPa}$, the rate of reduction gradually slows down.

3) The change of the porosity of the fracture structure will exist in two abruptly decreasing regions. The pore structure adjustment is related to the particle size gradation, and the reciprocal action between the water flow and the specimen will form a stable water conductivity channel at the inlet and outlet, and there are pores, collapses and cavities inside the filling body after infiltration.

4) The sudden water process triggered by mass loss has three stages. Particle loss under the sudden water process of the specimen can be divided into the initial seepage, disaster destabilization

380 and pipe flow water surge 3 stages, the flow rate and time relationship under each stage generally converge, the sudden water process will generally last $5 \sim 10$ s.

\section{Declarations}

383 The research study was carried out successfully with contribution from all authors, and all authors 384 approved the publication of the paper. The data used to support the findings of this study are available 
385 from the corresponding author upon request. The authors declare that there is no conflict of interest

386 regarding the publication of this paper. This work was supported by the National Natural Science 387 Foundations of China, Study on the Mechanism of Water-Gas Coupling Fracture Expansion and

388 Ultrasonic Characteristics of Coal Rock Mass in Drilling Holes (Grant No. 51774234); Fracture 389 evolution and water-gas coupled permeability mechanism of coal body around extraction borehole 390 (Grant No. 2021JM-390). The main research idea and manuscript preparation were contributed by 391 Mingkun Pang; Tianjun Zhang contributed on the manuscript preparation and performed the correlative 392 experiment. Rongtao Liu gave several suggestions from the industrial perspectives. Haotian Wang assisted on finalizing research work and manuscript. All research in the manuscript complies with ethical requirements and consent to participate. Finally, thanks to the test platform provided by Key Laboratory of Western Mine Exploitation and Hazard Prevention of the Ministry of Education, the test was successfully completed and the data was obtained.

\section{References}

[1] Wang T., Liu S., Wautier A., et al. (2021). An updated skeleton void ratio for gravelly sand mixtures considering the effect of grain size distribution[J]. Canadian Geotechnical Journal.

[2] Kong H., Wang L., Zhang H. (2020). The variation of grain size distribution in rock granular material in seepage process considering the mechanical-hydrological-chemical coupling effect: an experimental research[J]. Royal Society Open Science, 7(1): 190590.

412 [7] Kenney T., Lau D. (1986) Internal stability of granular filters: Reply[J]. Canadian Geotechnical

[3] Bai H., Ma D., Chen Z. (2013) Mechanical behavior of groundwater seepage in karst collapse pillars[J]. Engineering Geology, 164: 101-106.

[4] Peng S., Zhang J. (2007) Engineering Geology for Underground Rocks. Springer, Berlin.

[5] Wu J., Han G., Feng M., et al. (2019) Mass-loss effects on the flow behavior in broken argillaceous red sandstone with different particle-size distributions[J]. Comptes Rendus Mécanique, 347(6): 504-523.

[6] Zhao K., Xiong L., Kuang Z., et al. (2021) Uniaxial Compression Creep Characteristics and Acoustic Emission Characteristics of Two Different Kinds of Red Sandstone with Different Particle Sizes[J]. Journal, 22(3): 420-423. 
[8] Sterpi, Donatella. (2003) Effects of the Erosion and Transport of Fine Particles due to Seepage Flow[J]. International Journal of Geomechanics, 3(1): 111-122.

416 [9] Ma D., Duan H., Liu W., et al. (2020) Water-Sediment Two-Phase flow inrush hazard in rock fractures of overburden strata during coal mining[J]. Mine Water and the Environment.

418 [10] Yao B., Mao X., Wei J., et al. (2014) Study on coupled fluid-solid model for collapse columns considering the effect of particle transport[J]. Journal of China University of Mining \& Technology, 43(1): 30-35.

[11] Jie L., Chen Z., Gong X., et al. (2013) Test Method for the Determination of Soil Saturated Permeability Coefficients and the Optimization of Results[J]. Safety and Environmental Engineering.

[12] Ma D., Duan H., Li X., et al. Effects of seepage-induced erosion on nonlinear hydraulic properties

[13] Sulem J., Ouffroukh H. (2006) Hydromechanical Behaviour of Fontainebleau Sandstone[J]. Rock Mechanics \& Rock Engineering, 39(3): 185-213.

[14] Kong H., Chen Z., Wang L., et al. (2013) Experimental study on permeability of crushed gangues during compaction[J]. International Journal of Mineral Processing, 124 (Complete): 95-101.

[15] Yao B., Wei J., Wang D., et al. (2013) Numerical study on seepage property of karst collapse columns under particle migration[J]. Computer Modeling in Engineering and Sciences, 91(2): 81100.

[16] Zhang J. (2005) Investigations of water inrushes from aquifers under coal seams[J]. International Journal of Rock Mechanics and Mining Sciences, 42(3): 350-360.

[17] Yin S., Zhang J. (2005) Impacts of karst paleo-sinkholes on mining and environment in northern China[J]. Environmental Geology, 48(8): 1077-1083.

[18] Wan G., Yu J., Wang X., et al. (2021) Study on the pyrolysis behavior of coal-water slurry and coal-oil-water slurry.

[19] Zhu W., Wei C. (2011) Numerical simulation on mining-induced water inrushes related to geologic structures using a damage-based hydromechanical model[J]. Environmental Earth Sciences, 62(1): 43-54.

[20] Hui G., Yang Z., Han X. (2017) Study on reason for differences of hydraulic conductivity of karst collapse columns in Wanbei Mining Area[J]. Coal Science \& Technology. 
[21] Liu H., Li L., Li Z., et al. (2017) Numerical Modelling of Mining-induced Inrushes from Subjacent Water Conducting Karst Collapse Columns in Northern China[J]. Mine Water and the Environment.

[22] Xu Z., Sun Y., Gao S., et al. (2021) Comprehensive exploration, safety evaluation and grouting of karst collapse columns in the Yangjian coalmine of the Shanxi province, China[J]. Carbonates and Evaporites, 36(1).

[23] Zuo J., Hong Z., Peng S., et al. (2019) Investigation on failure behavior of collapse column in China's coal mine based on discontinuous deformation numerical method[J]. PLoS ONE, 14(8): e0219733.

[24] Fan Z. (2011) Research on characteristics and control technology of coal collapse column[J]. Coal Engineering.

[25] Sakthivadivel R. (1966) Theory and mechanism of filtration of noncolloidal fines through a porous medium[J]. University of California, Berkeley, HEL 15-25.

[26] Zhang J., Liu Y., Zhou N., et al. (2018) Pore pressure evolution and mass loss of broken gangue during the seepage[J]. Royal Society open science, 5(10): 180307.

[27] Elsworth D., Mao B. (1992) Flow-Deformation response of dual-porosity media[J]. Journal of Geotechnical Engineering, 118(1).

[28] Rubio-Marcos F., Romero J., Martín-Gonzalez M., et al. (2010) The mechanical properties of materials with interconnected cracks and pores[J]. International Journal of Rock Mechanics and Mining Sciences \& Geomechanics Abstracts, 33(5): 2763-2771.

[29] Pang M., Zhang T., Gao L., et al. (2022) Investigating the effects of effective stress on poredependent permeability measurements of crushed coal [J]. PLoS ONE.

[30] Somerton W., Sylemezolu I., Dudley R. (1975) Effect of stress on permeability of coal[J]. International Journal of Rock Mechanics \& Mining Sciences \& Geomechanics Abstracts, 12(5): $129-145$.

[31] Wang Z., Lin B., Yang W., et al. (2022) Fracture and pore development law of coal under organic solvent erosion[J]. Fuel, 307(6): 121815. 


\section{Supplementary Files}

This is a list of supplementary files associated with this preprint. Click to download.

- Declarationofinterests10.docx

- Highlights10.docx 